\title{
Recursos intangíveis e desempenho das exportações
}

Intangible resources and export performance

\section{Orlando Lima Rua}

Polytechnic of Porto (IPP), School of Accounting and Administration of Porto (ISCAP); Applied Management Research Unit (UNIAG); Center for Studies in Business and Legal Sciences (CECEJ), 4200-465 Porto, Portugal, orua@iscap.ipp.pt

\section{Alexandra França}

University of Minho, School of Economics and Management (EEC), Campus de Gualtar 4710-057 Braga, Portugal, franca.alexandra@gmail.com

\section{Resumo}

O objetivo fundamental deste estudo é analisar se os recursos intangíveis influenciam o desempenho das exportações.

Para tal, tendo em consideração a revisão da literatura sobre os temas estudados, realizamos uma investigação quantitativa, através de um questionário enviado para o responsável pelas atividades de exportação de pequenas e médias empresas (PME) portuguesas da indústria de calçado, associadas da Associação Portuguesa dos Industriais de Calçado, Componentes, Artigos em Pele e seus Sucedâneos (APICCAPS).

Confirmamos a positiva e significativa influencia que os recursos intangíveis têm no desempenho das exportações e que os recursos que mais contribuem para esse efeito são os recursos de reputação e o acesso aos recursos financeiros.

Este estudo apresenta os recursos intangíveis como um determinante estratégico do desempenho das exportações.

Palavras-chave: Recursos intangíveis, desempenho das exportações, PME, indústria portuguesa de calçado.

\section{Abstract}

The main goal of this study is to analyze if intangible resources influences export performance.

To this end, taking into account the literature review on the studied themes, we performed a quantitative research, through a survey sent to the responsible for the export activities of Small and Medium Sized Enterprises (SMEs) Portuguese footwear industry companies, associated of the Portuguese Footwear, Components and Leather Goods Association (APICCAPS).

We have confirmed the positive and significant influence that intangible resources carried out into export performance, and that the resources that most contribute to this end are reputation resources and access to financial resources.

This study presents intangible resources as a strategic determinant of export performance.

Keywords: Intangible resources, export performance, SMEs, Portuguese footwear industry.

\section{Introdução}

O novo padrão de competitividade obriga que o desempenho de uma empresa esteja relacionado com o valor dos recursos e competências, residindo o desafio da estratégia na seleção ou criação de um contexto ambiental onde as competências e recursos da empresa possam produzir vantagens competitivas (Porter \& Montgomery, 1998).

No âmbito internacional a sobrevivência e expansão das empresas, e o consequente crescimento económico dos países, está fortemente dependente de uma melhor compreensão dos determinantes estratégicos que influenciam o desempenho das exportações (Sousa, Martínez-López, \& Coelho, 2008). Desde os anos 90 que a concorrência implacável tem encaminhado as empresas para uma constante adaptação, renovação, reconfiguração e recriação dos seus recursos e capacidades de acordo com o ambiente competitivo (Wang \& Ahmed, 2007), sendo os recursos intangíveis considerados recursos estratégicos (Amit \& Schoemaker, 1993).

A Resourced-Based View (RBV) tornou-se numa teoria largamente estudada pelos académicos da gestão, em especial na investigação da performance das empresas, cujo referencial teórico é particularmente apropriado para estudar e clarificar os determinantes internos do desempenho das exportações (Morgan, Vorhies, \& Schlegelmilch, 2006; Sousa et al., 2008). Nesta teoria a dotação de recursos é um fator indispensável na explicação da heterogeneidade das empresas e permite compreender as diferentes estratégias escolhidas (Wang \& Ahmed, 2007).
Ao nível das empresas, através da diversificação do mercado, as exportações promovem oportunidades para que estas se tornem menos dependentes do mercado interno, conquistando novos clientes podem explorar economias de escala e alcançar custos de produção mais baixos enquanto produzem com mais eficiência (Okpara, 2009). Neste sentido, a exportação é uma das formas mais atrativas de entrar nos mercados externos, especialmente para as pequenas e médias empresas (PME), em comparação com as restantes alternativas, seja joint ventures ou a criação de subsidiárias, que implicam despender um elevado número de recursos (e.g. Dhanaraj \& Beamish, 2003; Piercy, Kaleka \& Katsikeas, 1998), não origina um grande risco e compromisso e permite uma maior flexibilidade ao ajustar o volume de bens exportados aos diferentes mercados internacionais (Lu \& Beamish, 2002).

A atividade exportadora de uma empresa é iniciada para satisfazer determinados objetivos, que podem ser económicos (como aumentar lucros e vendas) e/ou estratégicos (como a diversificação de mercados, ganhar quota nos mercados externos e aumentar reputação da marca) (Cavusgil \& Zou, 1994).

Por outro lado, as motivações da exportação podem resultar de ações proativas ou reativas. Nas ações proativas encontram-se a vantagem de lucro, a introdução de um produto único, a vantagem tecnológica, a informação exclusiva, o compromisso da gestão, os benefícios fiscais e as economias de escala. Ao nível das motivações reativas são identificadas as pressões competitivas, o excesso de capacidade produtiva, o decréscimo das vendas no mercado doméstico, a saturação do mercado doméstico e a 
proximidade dos clientes e dos portos de desembarque (Wood \& Robertson, 1997).

É essencial reconhecer e avaliar a importância dos recursos, uma vez que constituem fonte de vantagem competitiva quando permitem criar valor percebido pelo cliente, distinguir a empresa dos concorrentes, permitir competir em novos mercados e poderem ser usados numa ampla gama de produtos e serviços (Hamel \& Prahalad, 1994).

Este estudo pretende assim apoiar a formulação estratégica de políticas de gestão empresarial direcionadas para aumentar o desempenho das empresas nos mercados externos e acrescentar valor ao atual contexto de mudança. $O$ objetivo fundamental deste consubstancia-se assim em analisar se os recursos intangíveis influenciam o desempenho das exportações das PME portuguesas exportadoras da indústria do calçado, e de que forma tal influência se faz sentir.

\section{Revisão da Literatura}

\subsection{Recursos intangíveis}

Um princípio central da teoria baseada em recursos (ResourceBased Theory - RBT) é que as empresas com recursos valiosos, raros e insubstituíveis podem obter vantagens competitivas, pelo menos temporárias, usando esses recursos para desenvolver e implementar estratégias de produto-mercado (Hsu \& Ziedonis, 2013).

De facto, maior rentabilidade pode ser conseguida através da maximização da produtividade dos recursos. No entanto, os recursos não são, em si valiosos ou produtivos, mas permitem à empresa executar determinadas atividades em mercados específicos (Pearson, Pitfield, \& Ryley, 2015).

Existe consenso na literatura no sentido de que as fontes de vantagem competitiva estão bastante mais associadas aos recursos intangíveis do que aos tangíveis, uma vez que estes são mais raros e socialmente complexos, tornando a sua imitação difícil (e.g. Barney, 1991; Hitt, Bierman, Shimizu \& Kochhar, 2001).

Os recursos intangíveis são baseados no conhecimento ou informação, como a cultura organizacional, a reputação da marca, a própria marca da empresa, know-how, as suas capacidades são ilimitadas (Pearson et al., 2015) e têm uma gama muito mais ampla de utilizações nos mercados internacionais (Fernández-Olmos \& Díez-Vial, 2015).

Estes recursos podem ser mais facilmente reutilizados após uma mudança no mercado-alvo. Realmente as economias baseadas no conhecimento parecem ter melhorado muitos dos benefícios dos recursos intangíveis (Kozlenkova, Samaha, \& Palmatier, 2014).

Os recursos intangíveis têm três caraterísticas essenciais que os distinguem dos recursos tangíveis (Molloy, Chadwick, Ployhart, \& Golden, 2011). Primeiro, os intangíveis não se esgotam ou se deterioram com o uso, trazendo benefícios por um período de tempo indefinido e contrastando com os recursos tangíveis que têm a expetativa de desvalorização (Cohen, 2005). Em segundo lugar, vários gestores podem utilizar os intangíveis simultaneamente, por exemplo, o uso de uma marca está disponível para outros gerentes. Finalmente, os intangíveis são imateriais, tornando-os difíceis de comercializar, como a marca que não pode ser separada da organização (Marr \& Roos, 2005).

A literatura sugere que existem seis tipos de recursos intangíveis que constituem importante fonte de vantagem competitiva, particularmente nas empresas de exportação, são estes os recursos: a) de reputação; b) financeiros; c) humanos; d) culturais, e) relacionais; e f) de informação e conhecimento (Morgan et al., 2006).

Cada um destes recursos e o seu papel na determinação do desempenho das exportações são seguidamente testados e explicados.

\subsection{Desempenho das exportações}

As empresas, antes de operarem num mercado internacional, normalmente possuem informações imperfeitas sobre a oferta e a procura locais, regulamentos e requisitos jurídicos, bem como a adequação de seus produtos aos gostos e cultura locais. A vasta literatura no domínio dos negócios internacionais argumenta que a necessidade de aquisição de conhecimentos específicos do mercado externo obriga as empresas a seguirem um processo gradual de internacionalização, testando primeiro o mercado via exportações (Conconi, Sapir, \& Zanardi, 2016).

O desenvolvimento das exportações é de extrema importância, simultaneamente aos níveis macro e microeconómico. As exportações contribuem para o desenvolvimento económico e social das nações, ajudando as indústrias a desenvolver e melhorar a produtividade e criar empregos. Ao nível das empresas, através da diversificação do mercado, as exportações promovem uma oportunidade para que estas se tornem menos dependentes do mercado interno, conquistando novos clientes podem explorar economias de escala e alcançar custos de produção mais baixos enquanto produzem com mais eficiência (Okpara, 2009).

Neste sentido, a exportação é uma forma mais atrativa de entrar nos mercados externos, especialmente para as PME, em comparação com as restantes alternativas, seja joint ventures ou a criação de subsidiárias, que implicam despender um elevado número de recursos (Conconi et al., 2016; Dhanaraj \& Beamish, 2003; Piercy et al., 1998), não origina um grande risco e compromisso e permite uma maior flexibilidade ao ajustar o volume de bens exportados aos diferentes mercados internacionais (Lu \& Beamish, 2002).

A atividade exportadora de uma empresa é iniciada para satisfazer determinados objetivos, que podem ser económicos (como aumentar lucros e vendas) e/ou estratégicos (como a diversificação de mercados, ganhar quota nos mercados externos e aumentar reputação da marca) (Cavusgil \& Zou, 1994).

Por outro lado, as motivações da exportação podem resultar de ações proativas ou reativas. Nas ações proativas encontram-se a vantagem de lucro, a introdução de um produto único, a vantagem tecnológica, a informação exclusiva, o compromisso da gestão, os benefícios fiscais e as economias de escala. Ao nível das motivações reativas são identificadas as pressões competitivas, o excesso de capacidade produtiva, o decréscimo das vendas no mercado doméstico, a saturação do mercado doméstico e a proximidade dos clientes e dos portos de desembarque (Wood \& Robertson, 1997). 


\subsection{Relações entre os constructos}

A RBV defende que as variações na performance das empresas resultam da posse de recursos heterogéneos. Esta heterogeneidade de recursos e capacidades conduz a desequilíbrios do desempenho e afeta a habilidade das empresas em conceber e implementar estratégias competitivas (Barney, 1991; Peteraf, 1993). Assim, esta teoria afirma que a posse de recursos e capacidades heterogéneas afeta diretamente o desempenho das empresas (Makadok, 2001; Teece, Pisano, \& Shuen, 1997). Assim, ambicionamos testar as seguintes hipóteses:

H1: Os recursos intangíveis influenciam positiva e significativamente o desempenho das exportações.

H2: Os recursos humanos e culturais influenciam positiva e significativamente o desempenho das exportações.

H3: $O$ acesso aos recursos financeiros influenciam positiva e significativamente o desempenho das exportações.

H4: Os recursos de informação influenciam positiva e significativamente o desempenho das exportações.

H5: Os recursos de reputação influenciam positiva e significativamente o desempenho das exportações.

H6: Os recursos de relacionamento influenciam positiva e significativamente o desempenho das exportações.

\section{Metodologia de Investigação}

\subsection{Instrumento de medida e recolha de dados}

O instrumento utilizado foi o inquérito por questionário, no sentido em que não foi manipulada qualquer variável e todos os dados relacionados com as variáveis foram recolhidos ao mesmo tempo (Bryman \& Cramer, 2012). Este método de recolha de dados é mais adequados para este tipo de pesquisa (Sousa et al., 2008), uma vez que há a necessidade de interrogar um grande número de pessoas e existe um problema de representatividade (Campenhoudt \& Quivy, 2008).

O questionário é composto por vinte e seis questões fechadas, utilizando-se a escala de 5 pontos de Likert. As primeiras vinte e uma questões destinam-se a recolher informações acerca das capacidades absortivas e as seguintes a avaliar o desempenho das exportações, tendo sido elaborado com a utilização do limesurvey, versão 1.91 .

Com vista a reduzir situações incompreensão, o mesmo foi validado pelo gabinete de estudos da APICCAPS. De salientar que não foram detetadas dificuldades na interpretação das questões colocadas no questionário.

\subsection{Operacionalização e medida das variáveis}

A elaboração do modelo concetual sugerido implica o desenvolvimento de dimensões para cada um dos constructos. O modelo, apresentado contempla dois constructos: recursos intangíveis e desempenho das exportações.

\subsubsection{Recursos intangíveis}

Os recursos organizacionais podem ser entendidos como um conjunto de ativos tangíveis e intangíveis (Barney, Ketchen, \& Wright, 2011). Existe um consenso na literatura de que as fontes de vantagem competitiva estão muito mais associadas aos recursos intangíveis do que com os tangíveis. Para além disso, os ativos tangíveis tendem a depreciar no tempo, enquanto os ativos intangíveis podem acumular valor com o tempo (Porter, 1991). Assim, à luz da RBV, os recursos intangíveis constituem variáveis importantes para o sucesso da empresa (Amit \& Schoemaker, 1993; Barney, 1991).

Os recursos valiosos, raros, e inimitáveis devem ser definidos como variáveis exógenas e separadas das variáveis dependentes (Kozlenkova et al., 2014).

Para avaliação dos recursos de reputação utilizamos a escala proposta por Morgan et al. (2006). Pedimos aos inquiridos que avaliassem estes tipo de recursos da empresa disponíveis para o(s) mercado(s) de exportação, em relação aos seus principais concorrentes.

Esta dimensão é avaliada numa escala de Likert de cinco pontos, em que 1 "Muito Pior" e 5 "Muito Melhor".

\subsubsection{Desempenho das exportações}

A exportação é uma forma mais atrativa de entrar nos mercados externos, especialmente para as PME's, em comparação com as restantes alternativas, seja joint ventures ou a criação de subsidiárias, que implicam despender um elevado número de recursos (Conconi et al., 2016; Piercy et al., 1998).

Apesar de já terem sido realizados inúmeros estudos sobre a performance das empresas, não existe um consenso quanto ao instrumento de medida (Okpara, 2009). Este autor utilizou cinco variáveis para medir o constructo da performance: lucro, crescimento das vendas, crescimento no número de funcionários, expansão no mercado externo e a performance na generalidade (idem).

Assim, neste estudo utilizamos a escala de Okpara (2009), solicitando aos inquiridos para avaliar o seu desempenho no(s) mercado(s) de exportação em relação aos seus principais concorrentes. Os itens utilizados para medir o constructo do desempenho foram os indicadores de rentabilidade: crescimento das vendas, do lucro, das atividades e operações e da performance em geral.

Para medir cada um dos itens, foi utilizada a escala de Likert, com cinco níveis, onde 1 significa "discordo totalmente" e 5 "concordo totalmente".

\subsection{População, processo de recolha de dados e amostra}

Para a seleção da população fomos orientados pelo objetivo do presente estudo. Para testar a hipótese proposta no modelo operacional utilizamos uma amostra de empresas portuguesas, delimitando o nosso estudo empírico a empresas que cumulativamente obedecem aos seguintes critérios: fabricantes de calçado; empresas exportadoras; e capital social inteiramente nacional.

Esta escolha prende-se nomeadamente com: 1) as exportações são de extrema importância para a recuperação da crise económica e financeira que Portugal atravessa atualmente, tornando-se interessante estudar empresas exportadoras portuguesas; 2) a indústria do calçado é o sector que mais positivamente contribuiu para a balança comercial do país; e 3) as 
grandes multinacionais do calçado, que têm subsidiárias instaladas em Portugal, têm uma grande influência ao nível das estatísticas do comércio externo, sendo, neste sentido, interessante analisar o comportamento estratégico das empresas com capital social inteiramente nacional.

Para obter informação relativa às empresas estabelecemos contato com a APICCAPS a solicitar informação sobre as empresas exportadoras associadas. Foi-nos facultada uma base de dados com 231 empresas (nome da empresa, contato telefónico e correio eletrónico, CAE, mercados de exportação, intensidade de exportação e origem do capital). Destas apenas 167 empresas preenchiam os parâmetros estabelecidos. Cada uma destas empresas foi contatada por correio eletrónico, pela APICCAPS no sentido de as empresas responderem ao nosso questionário, com a hiperligação de acesso ao questionário, endereçado às pessoas que ocupavam cargos de gestão de topo e/ou de liderança da atividade de exportação.

A aplicação dos questionários decorreu entre 22 de abril e 22 de julho de 2014. Após finalizado o período de recolha de dados, foram recebidos 42 questionários válidos, correspondendo a uma taxa de resposta de $25 \%$, taxa bastante satisfatória atendendo ao facto de que a média de respostas dos gestores de topo ser entre 15\% e 20\% (Menon \& Bharadwaj, 1999).

Neste estudo optamos por uma amostra não probabilística e de conveniência, uma vez que os inquiridos foram escolhidos por serem associados da APICCAPS.

\section{Resultados}

Tabela 1 - Teste de consistência interna por dimensão (Alpha de Cronbach)

\begin{tabular}{|l|c|c|c|c|}
\multicolumn{1}{|c|}{ Dimensão } & $\boldsymbol{\alpha}$ de Cronbach & № de itens & $\mathbf{n}$ & Análise \\
\hline Recursos intangíveis & .963 & 23 & 42 & Excelente \\
\hline Desempenho das exportações & .927 & 5 & 42 & Excelente \\
\hline
\end{tabular}

Fonte: Elaboração própria.

Verificamos que todas elas apresentam valores de consistência excelentes.

\subsection{Análise fatorial exploratória}

Procedemos à análise fatorial de componentes principais (ACP), traduzindo-se esta num método de redução do número de variáveis correlacionadas, com um mínimo de perda de informação e nível de significância, num menor número de variáveis não correlacionadas que resumem a maior parte da informação presente nas variáveis originais (e.g. Hair, Black, Babin \& Anderson, 2009; Pestana \& Gageiro, 2008). Trata-se, no fundo, de renunciar a parte da informação, resumindo-a num novo e menor conjunto de variáveis agrupadas em fatores.

Após a extração dos fatores pela ACP, submetemos cada uma das extrações obtidas à rotação varimax, que permite obter fatores mais fáceis de interpretar e, teoricamente, com mais significado (Marôco, 2011).

A consistência interna dos fatores será analisada através dos respetivos Alphas de Cronbach e o teste KMO terão em consideração a utilização das escalas propostas por Pestana \& Gageiro (2008).

\subsection{Análise de fiabilidade}

Para verificarmos a fiabilidade da globalidade das variáveis estimamos a estabilidade e consistência interna através do Alfa de Cronbach $(\alpha)$. A consistência interna dos fatores define-se como a proporção da variabilidade nas respostas, as quais diferem devido à heterogeneidade das opiniões. De um modo geral, um instrumento ou teste é classificado como tendo fiabilidade apropriada quando o $\alpha$ é maior ou igual a 0.70 (Nunnally, 1978). Contudo, em alguns cenários de investigação das ciências sociais, um $\alpha$ de 0.60 é considerado aceitável desde que os resultados obtidos com esse instrumento sejam interpretados com precaução e tenham em consideração o contexto de computação do índice (DeVellis, 2012). Segundo Peterson (1994), este valor deve servir como uma base de partida e não como critério definitivo de classificação.

No entanto, existem pequenas divergências entre os diferentes autores relativamente à fiabilidade da consistência interna, sendo que para o presente estudo utilizamos a escala proposta por Pestana \& Gageiro (2008).

O resultado de 0.960 , obtido para a totalidade das variáveis do questionário, é considerado excelente, confirmando a consistência interna da amostra.

Na tabela 1 realizamos ainda o teste de consistência interna para o conjunto das variáveis que integram cada uma das dimensões, para assim avaliar a fiabilidade dos mesmos. 
e) Por fim, o quinto fator, denominado Recursos de Relacionamento, combina 4 itens, cujas saturações variam entre 0.800 e 0.607 .

Analisámos a consistência interna dos 5 fatores e verificámos que os alphas de cronbach são de $\alpha=0.943, \alpha=0.962, \alpha=0.882, \alpha=0.905$ e $\alpha=0.949$, respetivamente, para o 1.ㅇ, 2.ㅇ, 3.․, 4.ㅇ e 5.o fatores. Estes valores indicam que 4 das subdimensões apresentam uma excelente consistência interna e a quinta uma muito boa consistência interna.

O teste KMO indica-nos a existência de uma boa correlação entre as variáveis $(0,832)$.

Para o teste de esfericidade de Bartlett registou-se um valor de $\chi^{2}(253, \mathrm{n}=42)=1608.609, p<0.05$, logo consultando a tabela de distribuição de $\chi^{2}$ verifica-se que $\chi^{2}>\chi_{0.95}{ }^{2}$, pelo que se rejeita a hipótese nula, ou seja as variáveis estão correlacionadas.

\section{Desempenho das exportações}

No que respeita à análise fatorial do Desempenho das Exportações, foi extraído apenas um fator, não sendo necessário eliminar itens. Obtivemos uma escala composta por 5 itens, que explicam $77.90 \%$ da variância total.

$\mathrm{Na}$ estrutura fatorial da dimensão referente ao Desempenho das Exportações obtivemos 1 fator, designado da mesma forma, cujas saturações variam entre 0.850 e 0.918 .

Analisámos a consistência interna e verificámos que o Alpha de Cronbach é de $\alpha=0.927$. Este valor indica que esta dimensão apresenta uma excelente consistência interna. O teste KMO indica-nos a existência de uma boa correlação entre as variáveis (0.814). Para o teste de esfericidade de Bartlett registou-se um valor de $\chi^{2}(10, \mathrm{n}=42)=171.982, p<0.05$, logo consultando a tabela de distribuição de $\chi^{2}$ verifica-se que $\chi^{2}>\chi_{0.95}{ }^{2}$, pelo que se rejeita a hipótese nula, ou seja as variáveis estão correlacionadas.

\subsection{Regressão linear múltipla}

O modelo de Análise de Regressão Linear Múltipla é uma técnica estatística, descritiva e inferencial, que permite analisar as relações entre uma variável dependente e um conjunto de variáveis independentes (Pestana \& Gageiro, 2008). O termo variável dependente implica geralmente uma relação do tipo causa-efeito. Porém, a regressão linear pode ser usada para modelar a relação funcional entre duas variáveis, independentemente de existir ou não a relação tipo referida, que nem sempre é fácil de demonstrar (Marôco, 2011).

No modelo de regressão múltipla relacionamos duas variáveis através de um modelo linear, ou seja através da equação de uma reta do tipo $y=b_{0}+b_{1} x_{1}+\ldots+b_{k} x_{k}+\epsilon$, em que: $x_{1}, \ldots, x_{k}-$ variáveis explicativas ou independentes medidas sem erro (não aleatórias); $\epsilon-$ variável aleatória residual na qual se procuram incluir todas as influências no comportamento da variável y que não podem ser explicadas linearmente pelo comportamento das variáveis $x_{1}, \ldots, x_{k}$ e os possíveis erros de medição; $B_{0} \ldots B_{k}$ - parâmetros desconhecidos do modelo (a estimar); y - variável explicada ou dependente (aleatória) (Pestana \& Gageiro, 2008; Marôco, 2011). Os valores do eixo xi representam as variáveis independentes e são considerados determinísticos; habitualmente esta variável é designada por regressor. Os valores yi representam a variável dependente e estes são considerados variáveis aleatórias, conforme referido. Os coeficientes de reta são designados por coeficientes de regressão (Hall et al., 2011).

O valor indicado do coeficiente de determinação representado por $\mathrm{R}^{2}$ representa uma medida da dimensão do efeito das variáveis independentes sobre a variável dependente. Em regressão linear, este coeficiente é uma das estatísticas da qualidade de ajustamento mais populares. $O R^{2}$ mede a proporção da variabilidade total que é explicada pela regressão $(0 \leq R \leq 1)$. No caso das ciências sociais valores de $R^{2}>0.5$ consideram aceitável o ajustamento do modelo aos dados (Marôco, 2011).

Contudo, alguns autores sugerem que o valor de $\mathrm{R}^{2}$ não deve ser utilizado para comparar modelos que diferem relativamente ao número de variáveis dependentes diferentes, dado que geralmente a incorporação de mais uma variável independente tende a aumentar o $R^{2}$, mesmo que esta possua influência reduzida sobre a variável dependente (Marôco, 2011). Por outro lado, King (1986) refere a existência de um consenso de que não se pode avaliar a capacidade explicativa de um modelo de regressão a partir do $R^{2}$, devendo o foco da análise incidir na magnitude dos coeficientes e não na produção de um $R^{2}$.

No presente estudo, $R^{2}<0.5$, o que poderia sugerir um ajuste inadequado entre o modelo estimado e os dados observados. Não obstante, seguindo a recomendação de Figueiredo Filho, Nunes, Rocha, Santos, Batista, \& Silva Júnior (2011), os dados foram simulados para atender a todos os pressupostos do método de Mínimos Quadrados Ordinários (MQO), tendo assim as variáveis sido combinadas linearmente, destacando-se ainda o facto dos coeficientes estimados corresponderem aos valores estipulados na simulação. O foco residiu assim na interpretação dos coeficientes e não na produção de $\mathrm{R}^{2}$.

Para evitar que a utilização deste modelo de regressão pudesse produzir resultados incoerentes ou mesmo incorretos, dado compreender variáveis dependentes ordinais (Winship \& Mare, 1984), recorremos à regressão ordinal com vista a evitar a assunção de distâncias constantes entre as classes das variáveis (Long \& Freese, 2014).

Através da ANOVA da regressão, que compara a variância dentro das amostras ou grupos (também designada por variância residual, dos erros ou dentro dos grupos) com a variância entre as amostras ou grupos (também designada por variância do fator ou entre os grupos), podemos testar as hipóteses: HO: $\rho^{2}=0$ vs. $\mathrm{H} 1: \rho^{2} \neq 0$. De forma simplista, testamos o significado da regressão num todo, ou seja, testamos se tem significado considerar uma relação linear entre uma determinada variável e um conjunto de regressores.

A tabela 2 mostra para o modelo 1 , o qual se refere ao constructo dos recursos intangíveis, que $Z=2.431$, com um $\rho$-value $<0.1$ (Sig.), no liminar de $p<0.05$, pelo que podemos rejeitar $\mathrm{HO}$ em favor de H1. Para o modelo 3 obtivemos um valor de $Z=3.334$, com um $\rho$-value $=0.075(p<0.1)$, rejeitando-se ainda $\mathrm{HO}$ em favor de H3. O modelo 5 apresenta um valor de $Z=4.731$, com um $\rho$-value $=0.036$ $(p<0.05)$, rejeitando-se também $\mathrm{HO}$ em favor de H5. As hipóteses H1, H3 e H5 são suportadas, sendo as restantes, pelos resultados apresentados, não suportadas (H2, H4 e H6). 
Tabela 2 - ANOVA da regressão entre recursos intangíveis e desempenho das exportaçõesg

\begin{tabular}{|c|c|c|c|c|c|c|}
\hline Modelo & $\mathbf{R}$ & $\mathbf{R}^{\mathbf{2}}$ & $\mathbf{R}^{\mathbf{2}}$ Ajustado & $\begin{array}{c}\text { Erro padrão da } \\
\text { estimativa }\end{array}$ & $\mathbf{Z}$ \\
\hline $1^{\mathrm{a}}$ & $.502^{\mathrm{a}}$ & .252 & .149 & .92274230 & 2.431 \\
\hline $2^{\mathrm{b}}$ & $.178^{\mathrm{a}}$ & .032 & .007 & .99633358 & 1.302 & $.261^{* * *}$ \\
\hline $3^{\mathrm{c}}$ & $.277^{\mathrm{a}}$ & .077 & .054 & .97269805 & 3.334 \\
\hline $4^{\mathrm{d}}$ & $.072^{\mathrm{a}}$ & .005 & -.020 & 1.00981210 & $.075^{* *}$ \\
\hline $5^{\mathrm{e}}$ & $.325^{\mathrm{a}}$ & .106 & .083 & .95738806 & .207 & $.652^{* * *}$ \\
\hline $6^{\mathrm{f}}$ & $.182^{\mathrm{a}}$ & .033 & .009 & .99557699 & 1.365 \\
\hline
\end{tabular}

a. Preditores: (Constante), Recursos intangíveis.

b. Preditores: (Constante), Recursos humanos e culturais.

c. Preditores: (Constante), Acesso a recursos financeiros.

d. Preditores: (Constante), Recursos de informação.

e. Preditores: (Constante), Recursos de reputação.

f. Preditores: (Constante), Recursos de relacionamento.

g. Variável dependente: Desempenho das exportações.

$* p<0,05$.

** $p<0,1$.

*** n.s. - não significativo.

A simples comparação dos coeficientes de regressão para avaliar a importância de cada variável independente do modelo não é válida. Em primeiro lugar as variáveis independentes apresentam magnitudes diferentes. Assim, para que a importância das variáveis do modelo possam ser comparadas, é necessário usar variáveis standards no ajustamento do modelo ou então estandardizar os coeficientes de regressão, sendo estes designados por coeficientes Beta ( $\beta)$.
A tabela 3 mostra que as variáveis que apresentam maior contribuição para o comportamento das variáveis dependentes. Assim, podemos verificamos que os Recursos de Reputação $(\beta=0.325)$ e o Acesso a Recursos Financeiros $(\beta=0.470)$ são aqueles que mais potenciam o Desempenho das Exportações.

Tabela 3 - Coeficientes $\beta$ do modelo de regressão entre recursos intangíveis e desempenho das exportações

\begin{tabular}{|l|l|l|}
\hline \multicolumn{1}{|c|}{ Variáveis Incluídas } & & \multicolumn{1}{c|}{ Beta } \\
\hline RECURSOS INTANGÍVEIS & & \\
\hline Humanos e Culturais & .178 & $.226^{* * *}$ \\
\hline Acesso a recursos financeiros & .277 & $.062^{* *}$ \\
\hline Informação e conhecimento & .072 & $.621^{* * *}$ \\
\hline Reputação & .325 & $.030^{*}$ \\
\hline$<0,05$. & & \\
\hline $\begin{array}{l}0<0,1 . \\
\text { n.s. - não significativo. }\end{array}$ & \\
\hline
\end{tabular}

Fonte: Elaboração própria.

\section{Conclusões}

O objetivo fundamental deste estudo é analisar se os recursos intangíveis influenciam o desempenho das exportações.

Este estudo permitiu-nos concluir que os recursos intangíveis globalmente têm um impacto positivo e significativo no desempenho das exportações (H1 suportada) e que os recursos mais relevantes para esse efeito são os recursos de reputação (H3 suportada) e o acesso a recursos financeiros (H5 suportada).

A RBV defende que as variações no desempenho das empresas resultam da posse de recursos heterogéneos. Esta heterogeneidade de recursos e capacidades conduz a desequilíbrios do desempenho e afeta a habilidade das empresas em conceber e implementar estratégias competitivas (Barney, 1991; Peteraf, 1993). Assim, esta teoria afirma que a posse de recursos e capacidades heterogéneas afeta diretamente o desempenho das empresas (Makadok, 2001; Teece et al., 1997). Newbert (2007) sustenta que os recursos intangíveis, devido à sua inimitabilidade, são críticos para a obtenção de vantagem competitiva e que a posse destes representa também um fator importante na habilidade das PME implementarem estratégias que conduzam a resultados positivos ao nível do desempenho.
Os recursos de reputação estão relacionados com a imagem da empresa e podem ser fatores de diferenciação no mercado alvo (Hall, 1992). Estes recursos devem ser entendidos como fonte de vantagem competitiva, uma vez que são raros, de difícil imitação e transferência e permeiam a atividade da empresa (Barney, 1991). 0 recurso de reputação identificado na literatura como sendo o mais relevante para o desempenho das exportações é o valor da marca ou brand equity (Morgan et al., 2006). Estes recursos estão associados a um conjunto de ativos ligados ao nome e símbolo das marcas que acrescenta valor ao valor inicial do produto ou serviço, como a lealdade à marca, conhecimento do nome, qualidade percebida, associações à marca, entre outros (Aaker, 2010). Tal valioso recurso intangível permite à empresa exportadora construir e proteger a sua quota de mercado, potenciar os investimentos no marketing e lançar novos produtos no mercado de exportação alvo mais facilmente (Aaker, 2010).

As empresas com recursos financeiros tendem a responder mais rapidamente às mudanças do mercado e a obterem mais facilmente vantagem competitiva (Chesbrough \& Teece, 2002). O acesso ao capital permite às empresas aprender e superar desafios, bem como, investir em projetos de capital intensivo de forma a se protegerem nos mercados existentes ou entrarem em novos mercados (Westhead, Wright, \& Ucbasaran, 2001). Deste 
modo, o capital financeiro é um importante recurso, no entanto, a sua relevância está no seu acesso e não na sua propriedade (Wiklund \& Shepherd, 2005).

No contexto dos mercados internacionais as caraterísticas mais importantes dos recursos financeiros são o nível de financiamento que pode ser acedido e o momento em que pode ser utilizado (Morgan et al., 2006). Neste sentido, um melhor acesso a recursos financeiros aumenta a probabilidade de iniciar a exportação e reduzir o tempo de decisão de internacionalização (Bellone, Musso, Nesta, \& Schiavo, 2010).

A indústria portuguesa do calçado enfrenta desafios consideráveis, não apenas em relação à crise nos mercados internacionais, que restringe $o$ acesso a recursos financeiros, mas também em relação aos padrões de consumo. A redução dos ciclos de vida dos modelos de calçado tem consequências na oferta. Por um lado, os produtos têm que ser mais adaptados aos gostos e necessidades específicos dos diferentes segmentos (design personalizado, novos modelos em pequenas séries, etc.) e, por outro, as empresas devem apostar na marca, na imagem e no marketing para potenciar o lançamento de novos produtos.

\section{Implicações teóricas e práticas}

Deste estudo surgem importantes contributos para a teoria e prática, cujos resultados são relevantes para investigadores, gestores empresariais e entidades públicas e governamentais, dado consolidar a teoria baseada nos recursos (RBV) e especificamente os recursos intangíveis como um dos determinantes estratégicos do desempenho das exportações. Destaca-se assim o contributo deste estudo para a teoria da gestão estratégica, dado a estratégica abranger iniciativas deliberadas e emergentes adotadas pela gestão, compreendendo a utilização dos recursos e capacidades, para melhorar o desempenho das empresas (Nag, Hambrick \& Chen, 2007). Para se manterem competitivas, as empresas devem fazer uma avaliação interna com o objetivo de descobrir que recursos intangíveis lhes proporcionam vantagem em relação aos seus concorrentes por via de desempenhos superiores ao nível das suas exportações.

Os resultados obtidos são, portanto, um contributo para a clarificação da sua influência no desempenho das exportações das empresas. Adicionalmente, contribui para o desenvolvimento de conceitos, bem como para a definição e aplicação de escalas. A operacionalização dos constructos "recursos intangíveis" e "desempenho das exportações" permitiu a mensuração através de caraterísticas comuns e identificáveis entre as organizações.

O presente estudo possibilitou ainda uma análise aprofundada de uma indústria de elevada importância para as exportações nacionais, como é o caso da indústria do calçado, permitindo compreender quais as contribuições dos recursos intangíveis para o desempenho das exportações.

Tal terá implicações práticas, por um lado, para a gestão estratégica das empresas da referida indústria e, por outro, na (re)definição de políticas públicas de apoio e estímulo ao crescimento da atividade das respetivas empresas.

Neste estudo é assim notória, sobretudo, a importância dos recursos de reputação e do acesso aos recursos financeiros para o desempenho das exportações, sendo essencial que os empresários estejam aptos para a formulação de estratégias ajustadas às mudanças na procura, à busca de novas oportunidades de negócios e à otimização desses recursos nos seus processos.

\section{Limitações da investigação}

A principal limitação do presente estudo prende-se com a dimensão da amostra, uma vez que houve dificuldade em encontrar empresas com disponibilidade para colaborar neste tipo de investigação. $O$ facto desta ser não probabilística e por conveniência constitui também uma limitação. Pelo que se sugere prudência na generalização de resultados.

A maior parte das respostas ao questionário basearam-se no julgamento subjetivo dos respondentes. Apesar de se terem identificado as vantagens das medidas subjetivas para avaliar o desempenho das exportações, é possível que algumas respostas possam não representar a realidade do desempenho das empresas nos mercados externos.

Uma outra limitação reside no facto deste estudo não considerar o efeito das variáveis de controlo, tal como a dimensão, idade, localização e mercado alvo dos respondentes.

Por último, o facto do presente estudo considerar exclusivamente a forma de internacionalização referente à exportação pode ainda ser apontado como limitação.

\section{Linhas futuras de investigação}

Em trabalhos futuros, recomendamos que o modelo proposto seja usado numa amostra com um maior número de observações, para que seja possível confirmar os resultados obtidos. Por outro lado, sugere-se que se prossiga com a investigação da gestão estratégica em Portugal, incidindo também noutros setores da economia nacional, de modo a que, futuramente, se possa comparar o presente estudo com outros idênticos, permitindo assim perceber e encontrar novos fatores que contribuam para um melhor desempenho das exportações.

\section{Referências}

Aaker, D. A. (2010). Building Strong Brands. (Simon \& Schuster, Ed.). New York: Free Press.

Amit, R., \& Schoemaker, P. (1993). Strategic assets and organizational rent. Strategic Management Journal, 14(1), 33-46.

Barney, J. (1991). Firm Resources and Sustained Competitive Advantage. Journal of Management, 17(1), 99-120. doi:10.1177/014920639101700108

Barney, J., Ketchen, D. J., \& Wright, M. (2011). The Future of ResourceBased Theory: Revitalization or Decline? Journal of Management, 37(5), 1299-1315. doi:10.1177/0149206310391805

Bellone, F., Musso, P., Nesta, L., \& Schiavo, S. (2010). Financial Constraints and Firm Export Behaviour. The World Economy, 33(3), 347-373. doi:10.1111/j.1467-9701.2010.01259.x

Bryman, A., \& Cramer, D. (2012). Quantitative Data Analysis with IBM SPSS 17, 18 \& 19: A Guide for Social Scientists. Routledge.

Campenhoudt, L. Van, \& Quivy, R. (2008). Manual de investigação em Ciências Sociais. Gradiva Publicações.

Cavusgil, T., \& Zou, S. (1994). Marketing strategy-performance relationship: an investigation of the empirical link in export market ventures. Journal of Marketing, 58(1), 1-21.

Chesbrough, H., \& Teece, D. (2002). Organizing for innovation: when is virtual virtuous? The Innovative Enterprise, (August), 127-134.

Cohen, J. A. (2005). Intangible Assets: Valuation and Economic Benefit. Hoboken, NJ: Wiley. 
Conconi, P., Sapir, A., \& Zanardi, M. (2016). The internationalization process of firms: From exports to FDI. Journal of International Economics, 99, 16-30. doi:10.1016/j.jinteco.2015.12.004

DeVellis, R. F. (2012). Scale Development - Theory and Applications (3 $3^{\mathrm{a}} \mathrm{ed}$.). Los Angeles: SAGE Publications, Inc.

Dhanaraj, C., \& Beamish, P. W. (2003). A Resource-Based Approach to the Study of Export Performance. Journal of Small Business Management, 41(3), 242-261. doi:10.1111/1540-627X.00080

Fernández-Olmos, M., \& Díez-Vial, I. (2015). Intangible resources, export channel and performance: is there any fit? Journal of Business Economics and Management, 16(5), 1013-1033. doi:10.3846/16111699.2012.726928

Figueiredo Filho, D., Nunes, F., Rocha, E. C., Santos, M. L., Batista, M., \& Silva Júnior, J. A. (2011). O que Fazer e o que Não Fazer com a Regressão: pressupostos e aplicações do modelo linear de Mínimos Quadrados Ordinários (MQO). Revista Política Hoje, 20(1), 44-99.

Hair, J. F., Black, W. C., Babin, B. J., \& Anderson, R. E. (2009). Multivariate Data Analysis ( $7^{\mathrm{a}}$ ed.). Prentice Hall.

Hall, A., Neves, C., \& Pereira, A. (2011). Grande maratona de estatística no SPSS. Lisboa: Escolar Editora.

Hall, R. (1992). The strategic analysis of intangible resources. Strategic Management Journal, 13, 135-144.

Hamel, G., \& Prahalad, C. K. (1994). Competing for the Future. Harvard: Harvard Business Press.

Hitt, M., Bierman, L., Shimizu, K., \& Kochhar, R. (2001). Direct and moderating effects of human capital on strategy and performance in professional service firms: A resource-based perspective. Academy of Management Journal, 44(1), 13-28.

Hsu, D. H., \& Ziedonis, R. H. (2013). Resources as Dual Sources of Advantage: Implications for Valuing Entrepreneurial-Firm Patents. Strategic Management Journal, 34, 761-781.

King, G. (1986). How Not to Lie With Statistics: Avoiding Common Mistakes in Quantitative Political Science. American Journal of Political Science, 30, 666-687.

Kozlenkova, I. V., Samaha, S. A., \& Palmatier, R. W. (2014). Resource-based theory in marketing. Journal of the Academy of Marketing Science, 42(1), 1-21.

Long \& Freese (2014). Regression Models for Categorical Dependent Variables Using Stata, 3rd Edition, Texas: Stata Press.

Lu, J. W., \& Beamish, P. W. (2002). The Internationalization and Growth of SMEs. ASAC 2002, 86-96.

Makadok, R. (2001). Toward a synthesis of the resource-based and dynamic-capability views of rent creation. Strategic Management Journal, 22(5), 387-401.

Marôco, J. (2011). Análise estatística com o SPSS Statistics ( $5^{\mathrm{a}}$ ed.). Pêro Pinheiro: ReportNumber, Lda.

Marr, B., \& Roos, G. (2005). A strategy perspective on intellectual capital. In Perspectives on intelectual capital (pp. 28-52). Nova lorque: Routledge. Menon, a, \& Bharadwaj, S. (1999). Antecedents and consequences of marketing strategy making: A model and a test. Journal of Marketing, 63(April), 18-40.

Molloy, J. C., Chadwick, C., Ployhart, R. E., \& Golden, S. J. (2011). Making Intangibles "Tangible" in Tests of Resource-Based Theory: A Multidisciplinary Construct Validation Approach. Journal of Management, 37(5), 1496-1518. doi:10.1177/0149206310394185

Morgan, N., Vorhies, D. W., \& Schlegelmilch, B. B. (2006). Resourceperformance relationships in industrial export ventures: The role of resource inimitability and substitutability. Industrial Marketing Management, 35(5), 621-633. doi:10.1016/j.indmarman.2005.05.018

Nag, R., Hambrick, D., \& Chen, M. (2007). What is strategic management, really? Inductive derivation of a consensus definition of the field. Strategic Management Journal, 955(October 2006), 935-955. doi:10.1002/smj

Newbert, S. (2007). Empirical research on the resource-based view of the firm: an assessment and suggestions for future research. Strategic Management Journal, 146, 121-146. doi:10.1002/smj

Nunnally, J. C. (1978). Psychometric theory. New York: McGraw-Hill.

Okpara, J. (2009). Entrepreneurial orientation and export performance: evidence from an emerging economy. Int. Rev. Bus. Res. Papers, 5(6), 195-211. Pearson, J., Pitfield, D., \& Ryley, T. (2015). Intangible resources of competitive advantage: Analysis of 49 Asian airlines across three business models. Journal of Air Transport Management, 47, 179-189. doi:10.1016/j.jairtraman.2015.06.002

Pestana, M. H., \& Gageiro, J. N. (2008). Análise de Dados para Ciências Sociais - A complementaridade do SPSS ( $5^{\mathrm{a}}$ ed.). Lisboa: Edições Silabo.

Peteraf, M. (1993). The cornerstones of competitive advantage: A resource-based view. Strategic Management Journal, 14(3), 179-191.

Peterson, R. A. (1994). A Meta-Analysis of Cronbach's Coefficient Alpha. Journal of Consumer Research, 21(2), 381-391.

Piercy, N., Kaleka, A., \& Katsikeas, C. (1998). Sources of competitive advantage in high performing exporting companies. Journal of World Business, 33(4), 378-393.

Porter, M. (1991). Towards a dynamic theory of strategy. Strategic Management Journal, 12. doi:0143-2095/9 1/100095

Porter, M., \& Montgomery, C. A. (1998). Estratégia: a busca da vantagem competitiva. Rio de Janeiro: Campus.

Sousa, C. M. P., Martínez-López, F. J., \& Coelho, F. (2008). The determinants of export performance: A review of the research in the literature between 1998 and 2005. International Journal of Management Reviews, 10(4), 343374. doi:10.1111/j.1468-2370.2008.00232.x

Teece, D. J., Pisano, G., \& Shuen, A. (1997). Dynamic capabilities and strategic management. Strategic Management Journal, 18(7), 509-533. doi:10.1002/(SICI)1097-0266(199708)18:7<509::AID-SMJ882>3.0.CO;2-Z

Wang, C., \& Ahmed, P. K. (2007). Dynamic capabilities: A review and research agenda. International Journal of Management Reviews, 9(1), 3151. doi:10.1111/j.1468-2370.2007.00201.x

Westhead, P., Wright, M., \& Ucbasaran, D. (2001). The internationalization of new and small firms: A resource-based view. Journal of Business Venturing, 9026(99), 333-358.

Wiklund, J., \& Shepherd, D. (2005). Entrepreneurial orientation and small business performance: a configurational approach. Journal of Business Venturing, 20(1), 71-91. doi:10.1016/j.jbusvent.2004.01.001

Winship, C., \& Mare, R,D. (1984). Regression Models with Ordinal Variables. American Sociological Review, 49(August), 512-525.

Wood, V. R., \& Robertson, K. R. (1997). Strategic orientation and export success: an empirical study. International Marketing Review, 4(6), 424-444.

Recebido: 12.02.2016

Reenviado: 20.06 .2016

Aceite: 22.06 .2016 